\title{
Exact equivalent straight waveguide model for bent and twisted waveguides
}

\author{
Shyroki, Dzmitry
}

Published in:

IEEE Transactions on Microwave Theory and Techniques

Link to article, DOI:

10.1109/TMTT.2007.914637

Publication date:

2008

Document Version

Publisher's PDF, also known as Version of record

Link back to DTU Orbit

\section{Citation (APA):}

Shyroki, D. (2008). Exact equivalent straight waveguide model for bent and twisted waveguides. IEEE

Transactions on Microwave Theory and Techniques, 56(2), 414-419. https://doi.org/10.1109/TMTT.2007.914637

\section{General rights}

Copyright and moral rights for the publications made accessible in the public portal are retained by the authors and/or other copyright owners and it is a condition of accessing publications that users recognise and abide by the legal requirements associated with these rights.

- Users may download and print one copy of any publication from the public portal for the purpose of private study or research.

- You may not further distribute the material or use it for any profit-making activity or commercial gain

- You may freely distribute the URL identifying the publication in the public portal

If you believe that this document breaches copyright please contact us providing details, and we will remove access to the work immediately and investigate your claim. 


\title{
Exact Equivalent Straight Waveguide Model for Bent and Twisted Waveguides
}

\author{
Dzmitry M. Shyroki
}

\begin{abstract}
Exact equivalent straight waveguide representation is given for a waveguide of arbitrary curvature and torsion. No assumptions regarding refractive index contrast, isotropy of materials, or particular morphology in the waveguide cross section are made. This enables rigorous full-vector modeling of in-plane curved or helically wound waveguides with use of available simulators for straight waveguides without the restrictions of the known approximate equivalent-index formulas.
\end{abstract}

Index Terms-Coordinate transformation, equivalent straight waveguide, helical coordinates.

\section{INTRODUCTION}

$\mathbf{I}$ $\mathrm{N} 3-\mathrm{D}$ space, any curve can be defined by its curvature $\kappa$ and torsion $\tau$, which both may vary along the curve. If $\kappa$ and $\tau$ are constant, an ordinary helix is defined. In this paper, we shall consider electromagnetic waveguides whose axes are helical. Instead of specifying $\kappa$ and $\tau$, it is convenient to parameterize such waveguides by the pitch $2 \pi P$ and the radius, or axial shift $R$ (the latter introduced with some ambiguity), as in Fig. 1. By setting $P=0$, one arrives at an in-plane bend; by taking $R \rightarrow$ $\infty$, the waveguide gets straight.

Many specific full-vector methods to treat in-plane waveguide bends have been reported, most of them based on the separation of variables in cylindric coordinates and the subsequent treatment of waveguide modes in the cross section-by the source-type integral-equation technique [1], [2], the finitedifference [3]-[5] or finite-element [6] methods, the method of lines [7]-[9], etc. While being very efficient in terms of computation workload, these methods require substantial effort at the algorithm implementation stage, and cannot be used for modeling waveguide twists. More general are 3-D time-domain finite-difference [10], [11] and finite-element [12], [13] methods. Their common drawback is high computation cost: in one recent example [12], a 64-processor cluster was used for fiber bend propagation modeling.

A widely used approximate technique to treat waveguide bends is by introducing an equivalent straight waveguide with refractive index profile modified as [4], [14]-[17]

$$
n^{2} \approx \epsilon_{C}(1+\mathrm{y} / R)^{2} \approx \epsilon_{C}(1+2 \mathrm{y} / R) \approx \epsilon_{C} \exp (2 \mathrm{y} / R)
$$

Manuscript received August 2, 2007; revised November 18, 2007.

The author was with the Department of Communications, Optics and Materials, Technical University of Denmark, $2800 \mathrm{Kgs}$. Lyngby, Denmark. He resides in Minsk 220040, Belarus (e-mail: ds@com.dtu.dk).

Digital Object Identifier 10.1109/TMTT.2007.914637

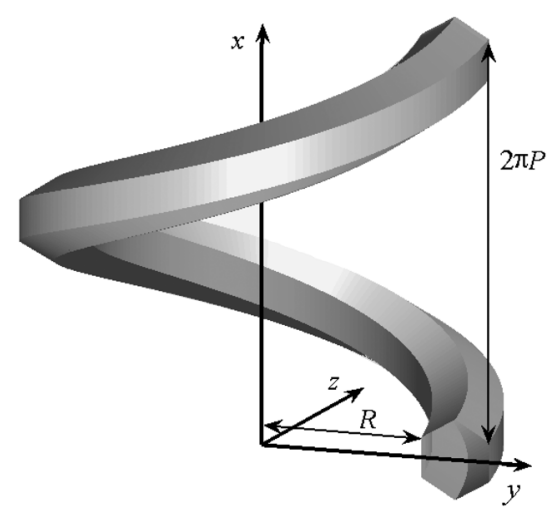

Fig. 1. Waveguide of arbitrary cross section wound in an helix. Two characteristic parameters are the pitch (or period) $2 \pi P$ and the radius $R$.

where $\epsilon_{C}=\epsilon_{C}(\mathrm{x}, \mathrm{y})$ is the dielectric permittivity (assumed scalar, as measured in a Cartesian frame) in the cross section perpendicular to the waveguide curved axis, $R$ is the bend radius, and $R+\mathrm{y}=y$ is the distance from the $x$-directed curvature axis. The equivalent-index approach is very appealing because there are many reliable tools for perfectly straight waveguides. Unfortunately, any of the equivalent-index formulas in (1) are only valid in the weak-guidance regime and for small curvature of an isotropic waveguide. Furthermore, none are useful in modeling twisted waveguides.

An interesting approach to modeling an homogeneously twisted waveguide with rigorous equivalent permittivity and permeability was reported in [18]. It was made possible due to the generally covariant formulation of Maxwell's equations, as sketched below in Section II. The drawback of the "rectangular helical" coordinate system used in [18] and [19] (in terminology of Waldron [20]) is that the waveguide is defined by its cross section in the $y z$-plane (see Fig. 1), which is convenient when the waveguide is twisted along its axis, but this prevents in-plane waveguide bend $(R \neq 0, P=0)$ being obtained by $P \rightarrow 0$. In contrast, the formulation in this paper is based on a "polar helical" system [20], or rather its modification specified in Section III. In Section IV, exact equivalent $\epsilon$ and $\mu$ profiles are derived, reducing to (1) when $P=0$ and the curvature is small. In Section V, two other important special cases are considered: an in-plane waveguide bend of arbitrary curvature, and a weakly twisted waveguide.

\section{MAXWELL'S EQUATIONS}

Owing to the generally covariant nature of classical electrodynamics [21], Maxwell's equations in arbitrary curvilinear co- 
ordinates can be written in a form identical to their Cartesian representation in a right-handed system as follows:

$$
\begin{aligned}
\mathfrak{E}^{i j k} \partial_{j} E_{k} & =-\mu^{i j} \dot{H}_{j} \\
\partial_{i} \mu^{i j} \dot{H}_{j} & =0 \\
\mathfrak{E}^{i j k} \partial_{j} H_{k} & =\epsilon^{i j} \dot{E}_{j}+\mathfrak{j}^{i} \\
\partial_{i} \epsilon^{i j} \dot{E}_{j} & =\rho
\end{aligned}
$$

where $\mathfrak{E}^{i j k}$ is the permutation tensor density, $\partial_{i}=\partial / \partial x^{i}$, time derivatives are denoted with dots, $i, j, k=1,2,3$, and the usual summation rule is assumed. The permittivity $\epsilon^{i j}$ and permeability $\mu^{i j}$ are tensor densities of weight +1 , which transform as

$$
\epsilon^{i^{\prime} j^{\prime}}=|\Delta|^{-1} J_{i}^{i^{\prime}} J_{j}^{j^{\prime}} \epsilon^{i j} \quad \mu^{i^{\prime} j^{\prime}}=|\Delta|^{-1} J_{i}^{i^{\prime}} J_{j}^{j^{\prime}} \mu^{i j}
$$

while $E_{i}$ and $H_{i}$ are covariant vectors

$$
E_{i^{\prime}}=J_{i^{\prime}}^{i} E_{i} \quad H_{i^{\prime}}=J_{i^{\prime}}^{i} H_{i}
$$

where $J_{i}^{i^{\prime}}=\partial_{i} x^{i^{\prime}}=J_{i}^{i^{\prime}}$ is the Jacobian transformation matrix, $\Delta=\operatorname{det} J_{i}^{i^{\prime}}$. For isotropic media possessing scalar permittivity $\epsilon_{C}$ and permeability $\mu_{C}$, measured in a Cartesian frame, transformation rules (3) reduce to

$$
\epsilon^{i^{i} j^{\prime}}=\sqrt{g} g^{i^{\prime} j^{\prime}} \epsilon_{C} \quad \mu^{i^{\prime} j^{\prime}}=\sqrt{g} g^{i^{\prime} j^{\prime}} \mu_{C}
$$

owing to the transformation behavior of the metric tensor $g^{i^{\prime} j^{\prime}}=J_{i}^{i^{\prime}} J_{j}^{j^{\prime}} g^{i j}$ and insofar as $g^{i j}=\delta_{i j}$ in a Cartesian frame. The determinant of covariant metric $g=\operatorname{det} g_{i^{\prime} j^{\prime}}=\Delta^{-2}$, to comply with standard notation. Note also that the "relative" and "free-space" permittivity and permeability are often used such that $\epsilon=\epsilon_{r} \epsilon_{0}$ and $\mu=\mu_{r} \mu_{0}$, and the free-space speed of light $c=1 / \sqrt{\epsilon_{0} \mu_{0}}$.

Since Maxwell's equations (2) are indistinguishable from those for Cartesian components of three-vectors $\mathbf{E}$ and $\mathbf{H}$, an ordinary mode solver based on a Cartesian grid can be employed for any coordinate system, given the permittivity and permeability profiles modified via (3) or (5). Cartesian components of "conventional" three-vectors $\mathbf{E}$ and $\mathbf{H}$ can then be restored from $E_{i}$ and $H_{i}$ via (4) if necessary. Furthermore, if the transformed $\epsilon$ and $\mu$ appear to be independent on one of the coordinates, standard separation of variables becomes possible.

\section{HELICAL COORDINATES}

Several coordinate systems were used with helical structures. In [18] and [19] and some earlier works on waveguides twisted along their axes, coordinates $\mathbf{x}^{\prime}=(u, v, w)$ were used related to the Cartesian system $\mathbf{x}=(x, y, z)$ by

$$
\begin{aligned}
& x=u \\
& y=v \cos (u / P)+w \sin (u / P) \\
& z=-v \sin (u / P)+w \cos (u / P) .
\end{aligned}
$$

The corresponding distance element is

$$
\begin{array}{r}
\mathrm{d} s^{2}=\left[1+(v / P)^{2}+(w / P)^{2}\right] \mathrm{d} u^{2}+\mathrm{d} v^{2} \\
+\mathrm{d} w^{2}+2(w / P) \mathrm{d} u \mathrm{~d} v-2(v / P) \mathrm{d} u \mathrm{~d} w .
\end{array}
$$

Hence, the metric tensor does not depend on $u$ and, thus, the permittivity and permeability transformed according to (5) do not acquire dependence on $u$ for a waveguide defined by its cross section in the $y z$-plane and by a constant rate of rotation around the $x$-axis. As mentioned in Section I, however, this transformation is not meaningful for $P=0$.

A different transformation from Cartesian to the "polar helical" system (we denote new coordinates by $u, v, w$ as before) was proposed in [20] as follows:

$$
\begin{aligned}
& x=u+P \phi \\
& y=v \cos \phi \\
& z=v \sin \phi
\end{aligned}
$$

with $\phi=w / R$. (We use the longitudinal helical coordinate $w=R \phi$ instead of the conventional polar coordinate $\phi$, and denote the radial coordinate by $v$ for coherent notation in this paper. The choice was made to ease use of the equivalent-index formulas below.) The corresponding distance element

$$
\mathrm{d} s^{2}=\mathrm{d} u^{2}+\mathrm{d} v^{2}+\left[(v / R)^{2}+(P / R)^{2}\right] \mathrm{d} w^{2}+2(P / R) \mathrm{d} u \mathrm{~d} w
$$

shows that the metric tensor depends solely on $v$ now and parametrically on $R$ and $P$ with components $g_{12}=g_{21}=0$ and $g_{13}=g_{31}=$ const, unlike in (7), and it reduces to the metric of cylindric system by setting $P=0$. We may thus use transformation (8) for our purposes. It is still not very practical, however, as far as the waveguide must be defined by its $(x, y)$ cross section, which is not perpendicular to the waveguide axis once $P \neq 0$. We follow [22] to modify (8) as follows:

$$
\begin{aligned}
& x=P_{u} \phi_{u}+P_{w} \phi_{w} \\
& y=v \cos \left(\phi_{w}-\phi_{u}\right) \\
& z=v \sin \left(\phi_{w}-\phi_{u}\right)
\end{aligned}
$$

with $\phi_{u}=u / \sqrt{R^{2}+P_{u}^{2}}$ and $\phi_{w}=w / \sqrt{R^{2}+P_{w}^{2}}$. The $u$ and $w$ coordinate lines are helices of opposite handedness. It is easy to see that with $P_{u} \rightarrow \infty$, the transformation (10) is reduced to (8). In the following, we find it convenient to impose orthogonality between the two families of helices at $v=R$ by choosing $P_{u}$ such that $P_{u} P_{w}=R^{2}$; the values for $P_{w} \equiv P$ and $R$ are obviously dictated by waveguide geometry. Subject to such constraint, $u$ in (10) can be called the transverse helical coordinate. The distance element

$$
\begin{aligned}
\mathrm{d} s^{2}=\frac{(v / R)^{2}+(R / P)^{2}}{1+(R / P)^{2}} \mathrm{~d} u^{2}+ & \mathrm{d} v^{2}+\frac{(v / R)^{2}+(P / R)^{2}}{1+(P / R)^{2}} \mathrm{~d} w^{2} \\
& +2 \frac{1-(v / R)^{2}}{R / P+P / R} \mathrm{~d} u \mathrm{~d} w
\end{aligned}
$$


defines the components of covariant metric tensor in the $u, v, w$ coordinates given by (10) with $P_{u}=R^{2} / P$. It is seen that $g=v^{2} / R^{2}$ and the contravariant tensor that occurs in (5) is

$$
g^{i^{\prime} j^{\prime}}=\left[\begin{array}{ccc}
\frac{1+(P / v)^{2}}{1+(P / R)^{2}} & 0 & \frac{1-(R / v)^{2}}{R / P+P / R} \\
0 & 1 & 0 \\
\frac{1-(R / v)^{2}}{R / P+P / R} & 0 & \frac{(R / v)^{2}+(P / R)^{2}}{1+(P / R)^{2}}
\end{array}\right] .
$$

Another helical coordinate system proposed by Germano in [23] is strictly orthogonal (not merely at $v=R$ ), but it does not retain invariance along the generating helix and, thus, makes separation of variables impossible.

\section{EQUivalent PRofiles}

\section{A. Isotropic Waveguides}

One may use (5) with the contravariant metric tensor given by (12) to obtain $\epsilon$ and $\mu$ in the new coordinates for a bent and twisted waveguide made of isotropic materials. The expressions for curvature and torsion of an ordinary helix

$$
\kappa=\frac{R}{R^{2}+P^{2}} \quad \tau=\frac{P}{R^{2}+P^{2}}
$$

allow to write $\epsilon$ transformed via (5) as follows:

$$
\begin{aligned}
\epsilon^{u u} / \epsilon_{C} & =v \kappa+\left[v \kappa\left(\frac{\kappa}{\tau}+\frac{\tau}{\kappa}\right)^{2}\right]^{-1} \\
\epsilon^{v v} / \epsilon_{C} & =v \kappa+\frac{v \tau^{2}}{\kappa} \\
\epsilon^{w w} / \epsilon_{C} & =\frac{v \tau^{2}}{\kappa}+\left[\frac{v \tau^{2}}{\kappa}\left(\frac{\kappa}{\tau}+\frac{\tau}{\kappa}\right)^{2}\right]^{-1} \\
\epsilon^{u w} / \epsilon_{C} & =v \tau-\left[v \tau\left(\frac{\kappa}{\tau}+\frac{\tau}{\kappa}\right)^{2}\right]^{-1}
\end{aligned}
$$

and similarly for $\mu$. Given the curvature and torsion, one has to modify $\epsilon$ according to (14) and $\mu$ analogously, and to employ any conventional algorithm to solve Maxwell's equations (2) for a straight waveguide. We, therefore, call (14) and analogous expressions for the $\mu$ components the equivalent straight waveguide profiles. In the new coordinates, the curved waveguide is $w$-invariant ("straight"), defined by its cross section in the $u v$ coordinate surface. It should be noted that this is a helical surface defined by $w=$ const in (10), not a plane. It is close to a plane perpendicular to the axis of a waveguide whose width is small enough compared to the torsion reciprocal $\delta v \ll \tau^{-1}$, but care should be taken when defining the $w=$ const cross section of a highly twisted waveguide. The condition $\delta v \ll \tau^{-1}$ does not limit the validity of (14); however, it is just a matter of convenience in defining waveguide geometry.

An interesting observation follows from (14), or rather from (12) already: due to the independence of the metric tensor on the transverse helical coordinate $u$, and its off-diagonal components — which reverse their sign under the $u$-reflection-vanishing at the radial coordinate $v$ being close to $R$, separation of waveguide modes into TE and TM classes occurs in a narrow $\left(\delta v \ll \tau^{-1}\right.$ ) waveguide symmetric with respect to some $u=$ const surface. Such separation is not seen in the standard "polar helical" coordinates (8) or "rectangular helical" system (6) since $\epsilon^{u w}$ and $\mu^{u w}$ are essentially nonzero in those frames. A related observation regards waveguide reversibility: with nonzero $\epsilon^{u w}$ and $\mu^{u w}$, even the waveguide perfectly homogeneous along $w$ is not invariant under $w$-reflection in the $u, v, w$ coordinates. Yet, again, it is almost reciprocal if sufficiently narrow.

\section{B. Anisotropic Waveguides}

Formulas (14a)-(14d), derived via (5), are applicable only to a waveguide made of isotropic materials. Often, the effects of anisotropy should be taken into account. This can be achieved easily by using the general transformation rules (3) and noting that the transformation matrix for contravariant components, as obtained from (10), can be conveniently decomposed as follows:

$$
J_{i}^{i^{\prime}}=\left[\begin{array}{ccc}
\frac{1}{\sqrt{1+(P / R)^{2}}} & 0 & \frac{-P / v}{\sqrt{1+(P / R)^{2}}} \\
0 & 1 & 0 \\
\frac{P / R}{\sqrt{1+(P / R)^{2}}} & 0 & \frac{R / v}{\sqrt{1+(P / R)^{2}}}
\end{array}\right] \mathcal{R}_{u} \mathcal{R}_{w}^{-1}
$$

where

$$
\mathcal{R}_{i}=\left[\begin{array}{ccc}
1 & 0 & 0 \\
0 & \cos \phi_{i} & -\sin \phi_{i} \\
0 & \sin \phi_{i} & \cos \phi_{i}
\end{array}\right]
$$

Since we consider $w$-invariant waveguides, such that the permittivity measured in a Cartesian frame along some $w=$ const surface is $\epsilon_{C}=\left.\mathcal{R}_{w} \epsilon_{C}\right|_{w=0} \mathcal{R}_{w}^{-1}$ and similarly for $\mu_{C}$, the $\mathcal{R}_{w}$ matrices cancel out in $\epsilon$ and $\mu$ transformed according to (3) as follows:

$$
\begin{aligned}
\epsilon=\frac{v / R}{1+(P / R)^{2}}\left[\begin{array}{ccc}
1 & 0 & -P / v \\
0 & \sqrt{1+(P / R)^{2}} & 0 \\
P / R & 0 & R / v
\end{array}\right] \\
\quad \times\left.\mathcal{R}_{u} \epsilon_{C}\right|_{w=0} \mathcal{R}_{u}^{-1}\left[\begin{array}{ccc}
1 & 0 & P / R \\
0 & \sqrt{1+(P / R)^{2}} & 0 \\
-P / v & 0 & R / v
\end{array}\right]
\end{aligned}
$$

and similarly for $\mu$. Now, unlike in the case of isotropic waveguides (14), the equivalent profiles depend on the sine and cosine functions of $u$ in $\mathcal{R}_{u}$ 's as far as $P \neq 0$.

\section{Numerical Implementation}

The continuous dependence in (14) on the radial coordinate $v$ or in (15) on both $u$ and $v$ prohibits use of integral-equation methods to solve the dimensionality reduced Maxwell's equations with (14) or (15). We are thus concerned with the implementation of (14) and (15) in the finite-difference or finite-element simulations.

The presence of nonzero off-diagonal components in (14) and (15) has several consequences. First, Yee's scheme [24]-a popular field staggering scheme in the finite-difference time-domain and frequency-domain computations-becomes not very well suited. It should be augmented with some averaging of the off-diagonal components over neighboring grid nodes [25] or changed to the collocated scheme [26]. Second, nonreciprocity 
in the $w$-direction may complicate some algorithms: e.g., both odd and even terms in the propagation constant $\beta$ would appear in the frequency-domain Maxwell's equations, thus prohibiting their formulation as an ordinary $\beta$ eigenproblem. Finally, the perfectly matched layers (PMLs) used commonly to represent open boundaries in the finite-difference or finite-element calculations [27] should be constructed as shown below.

The PML method is similar to the complex-coordinate transformation technique in the quantum theory of atomic resonances [28]. Within the electromagnetics modeling community, the relation between the complex-valued coordinate $x^{1}$ and the real-valued bounded computational coordinate $x^{1^{\prime}}$ is commonly written as

$$
x^{1}=\int_{0}^{x^{1^{\prime}}} s_{1}\left(x^{1^{\prime}}, \omega\right) \mathrm{d} x^{1^{\prime}} \quad s_{1}\left(x^{1^{\prime}}, \omega\right)=1+i \frac{\sigma\left(x^{1^{\prime}}\right)}{\omega}
$$

where the "coordinate-stretching" function $s_{1}$ is the Jacobian matrix component, $s_{1}=\partial x^{1} / \partial x^{1^{\prime}}=J_{1^{\prime}}^{1}$, and $\sigma\left(x^{1^{\prime}}\right)$ defines the PML conductivity profile. Treating (16) as an ordinary transform of the "first" coordinate of a given (not necessarily Cartesian) coordinate system, one can modify $\epsilon$ and $\mu$ in the PML regions as [28]

$$
\hat{\epsilon}=\left[\begin{array}{ccc}
1 & 0 & 0 \\
0 & s_{1} & 0 \\
0 & 0 & s_{1}
\end{array}\right] \epsilon\left[\begin{array}{ccc}
s_{1}^{-1} & 0 & 0 \\
0 & 1 & 0 \\
0 & 0 & 1
\end{array}\right]
$$

and similarly for $\hat{\mu}$. Constructing the PMLs in the transverse helical $\left(x^{1}=u\right)$ and radial $\left(x^{2}=v\right)$ directions of our $u, v, w$ coordinates leads to

$$
\hat{\epsilon}=\left[\begin{array}{ccc}
s_{v} & 0 & 0 \\
0 & s_{u} & 0 \\
0 & 0 & s_{u} s_{v}
\end{array}\right] \epsilon\left[\begin{array}{ccc}
s_{u}^{-1} & 0 & 0 \\
0 & s_{v}^{-1} & 0 \\
0 & 0 & 1
\end{array}\right] .
$$

In the following examples, we consider isotropic waveguides described by the equivalent profiles (14) so we rewrite (17) for such a case explicitly as follows:

$$
\begin{aligned}
\hat{\epsilon}^{u u} & =\epsilon^{u u} s_{v} / s_{u} \\
\hat{\epsilon}^{v v} & =\epsilon^{v v} s_{u} / s_{v} \\
\hat{\epsilon}^{w w} & =\epsilon^{w w} s_{v} s_{u} \\
\hat{\epsilon}^{u w} & =\epsilon^{u w} s_{v}
\end{aligned}
$$

while the $v$ coordinate in (14) is transformed via (16). It must be remembered that the magnetic permeability should be treated in the same way even when $\mu_{r C}=1$.

\section{Special CASES}

\section{A. $\tau=0, \kappa \neq 0$}

An important special case, which we will explicitly consider first, is an in-plane waveguide bend, i.e., $\tau=0$ and, hence, $\kappa=R^{-1}$. Formulas (14a)-(14d) are reduced to

$$
\epsilon^{u u}=\epsilon^{v v}=\epsilon_{C} v / R \quad \epsilon^{w w}=\epsilon_{C} R / v \quad \epsilon^{u w}=0 .
$$

Exactly the same formulas follow from [29, eq. (12)] specified for isotropic $\epsilon_{C}$. These formulas were tested extensively, and no numerical problems in the vicinity of the $v=0$ axis were observed. To clearly see the relation of (19) with approximate equivalent index expressions (1), we shift the radial coordinate $\mathrm{y}=v-R$ and designate $u=\mathrm{x}$ and $w=\mathrm{z}$. We thus get

$$
\epsilon^{\mathrm{xx}}=\epsilon^{\mathrm{yy}}=\epsilon_{C}(1+\mathrm{y} / R) \quad \epsilon^{\mathrm{zz}}=\epsilon_{C}(1+\mathrm{y} / R)^{-1} .
$$

The expressions for $\epsilon^{\mathrm{xx}}$ and $\epsilon^{\mathrm{yy}}$, multiplied by their $\mu$-counterparts, lead to refractive tensor components $n_{\mathrm{xx}}^{2}=\epsilon^{\mathrm{xx}} \mu^{\mathrm{xx}}$ and $n_{\mathrm{yy}}^{2}=\epsilon^{\mathrm{yy}} \mu^{\mathrm{yy}}$, which are precisely in line with (1) in the case of nonmagnetic media. The $\epsilon^{\mathrm{zz}}$ and $\mu^{\mathrm{zz}}$ components manifestly differ from the rest, however. This difference is negligible for low-contrast slightly bent waveguides, but comes into play upon departing from the weakly guiding regime, when significant $z$-components of electric and magnetic fields start "probing" the $\epsilon^{\mathrm{zz}}(\mathrm{x}, \mathrm{y})$ and $\mu^{\mathrm{zz}}(\mathrm{x}, \mathrm{y})$ profiles.

Our first illustration to the validity of (19), or (20), is the problem of light propagation in a slab waveguide bend amenable to analytic treatment [30]. After substituting (19) and similar formulas for $\mu$ into the frequency-domain Maxwell's curl equations and setting $\partial_{u}=0$ and $\partial_{w}=i \beta$, one gets two pairs of first-order scalar equations: one pair for the $E_{u}$ and $H_{w}$ components (TE mode, in the infinite slab nomenclature) as follows:

$$
\begin{aligned}
\partial_{v} E_{u} & =-i \omega \frac{\mu_{C} R}{v} H_{w} \\
\partial_{v} H_{w} & =-i \omega\left(\frac{\epsilon_{C} v}{R}-\frac{\beta^{2} R}{\omega^{2} \mu_{C} v}\right) E_{u}
\end{aligned}
$$

and another pair for $E_{w}$ and $H_{u}$ (TM mode). To derive a secondorder equation for $E_{u}$, for example, one combines the above two equations to get, in the regions of constant $\mu_{C}$ the following:

$$
\frac{\partial^{2} E_{u}}{\partial v^{2}}+\frac{1}{v} \frac{\partial E_{u}}{\partial v}+k^{2}\left(\epsilon_{r C} \mu_{r C}-\frac{n_{\text {eff }}^{2} R^{2}}{v^{2}}\right) E_{u}=0
$$

where $n_{\text {eff }}=\beta / k$ and $k=\omega / c$. This is a well-known Bessel equation for TE modes in a bent slab waveguide [30], while an attempt to get the same equation with approximate equivalentindex formulas in (1) fails.

The second example numerically demonstrates the failure of (1) at tight bends as those analyzed in [6] for a silicon waveguide of rectangular $0.445 \mu \mathrm{m} \times 0.220 \mu \mathrm{m}$ cross section lying on a plane silica substrate. The waveguide bend radius is taken to be $2 \mu \mathrm{m}$. In Fig. 2, we reproduced [6, Fig. 3(b)] with use of equivalent profile methods, the here proposed exact and the standard approximate one. Unlike in [6], featuring special finite-element implementation for cylindric coordinates, here we used an usual compact 2-D full-vector finite-difference frequency-domain (FDFD) method: the frequency-domain Maxwell's equations were discretized according to the staggered 2-D (projected) Yee scheme, and the resulting ordinary eigenproblem in the waveguide mode index $n_{\text {eff }}$ solved iteratively in MATLAB with the eigs function. Noteworthy, calculations on a $80 \times 80$ grid took a few seconds per wavelength point on a 1.6-GHz Intel Pentium M laptop computer-compare against $50 \mathrm{~s}$ reported in [6] for the finite-element modeling in cylindric coordinates. This difference in timing can be attributed partly to the FDFD 


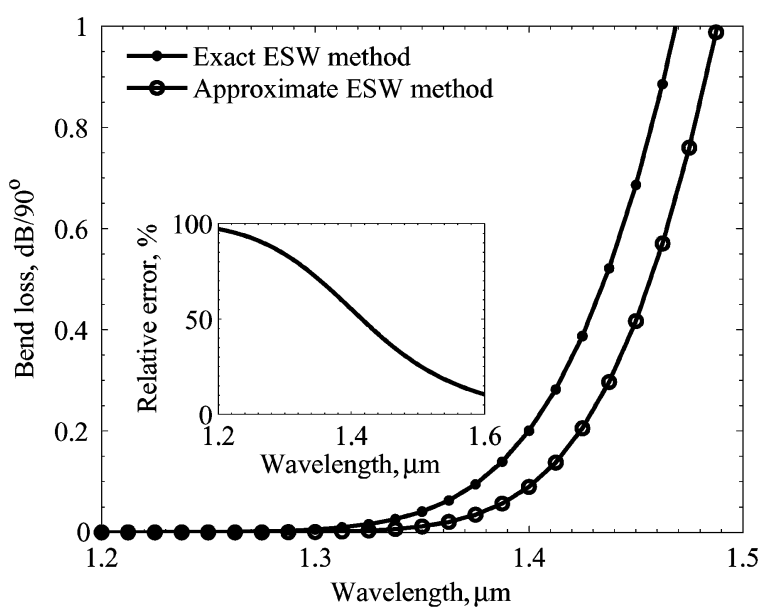

Fig. 2. Bend losses per turn $\left(90^{\circ}\right)$ for a quasi-TM mode in an $R=2 \mu \mathrm{m}$ bent, $0.445 \mu \mathrm{m} \times 0.22 \mu \mathrm{m}$ waveguide as modeled in [6]. The refractive indices are $n_{\text {core }}=3.5, n_{\text {clad }}=1.45$, and $n_{\text {air }}=1.00$. For the finite-difference modeling, an $80 \times 80$ grid was used. For each wavelength value, it took a few seconds on a 1.6-GHz Intel Pentium M laptop computer to find the mode.

method being faster than the finite-element method (FEM), as such, and partly by the simplicity and, hence, efficiency of the logically Cartesian algorithms.

Generalization of (20) for an in-plane bend of an arbitrary anisotropic waveguide, if only invariant under rotations around the curvature axis, $\epsilon_{C}(\mathrm{x}, \mathrm{y}, \mathrm{z})=\left.\mathcal{R}_{\mathrm{z}} \epsilon_{C}\right|_{\mathrm{z}=0}(\mathrm{x}, \mathrm{y}) \mathcal{R}_{\mathrm{z}}^{-1}$, is easy to obtain from the general formula (15) as follows:

$$
\epsilon=\left.\left[\begin{array}{ccc}
1+\frac{\mathrm{y}}{R} & 0 & 0 \\
0 & 1+\frac{\mathrm{y}}{R} & 0 \\
0 & 0 & 1
\end{array}\right] \epsilon_{C}\right|_{\mathrm{z}=0}\left[\begin{array}{ccc}
1 & 0 & 0 \\
0 & 1 & 0 \\
0 & 0 & \frac{1}{1+\frac{\mathrm{y}}{R}}
\end{array}\right]
$$

and similarly for $\mu$. As in the case of an isotropic waveguide bend, this allows mode separation in the TE and TM types for x-symmetric $\epsilon_{C}$ and $\mu_{C}$, and reversibility for z-invariant $\epsilon_{C}$ and $\mu_{C}$ with no $\mathrm{xz}$ or yz components.

B. $\tau \approx \kappa \ll \delta v^{-1}$

We have two reasons in this case to omit off-diagonal components $\epsilon^{u w}$ and $\mu^{u w}$. First, when the torsion and the curvature of a waveguide are comparable one to another by an order of magnitude while the waveguide is sufficiently narrow, i.e., $\delta v \ll R \approx P$, one obtains from (14), or from (12) already

$$
\epsilon^{u w} / \epsilon^{u u} \approx \epsilon^{u w} / \epsilon^{v v} \approx \epsilon^{u w} / \epsilon^{w w} \ll 1 .
$$

Second, in a weakly guiding dielectric waveguide, the electromagnetic modes are predominantly transversal and, thus, are sensitive to the $\epsilon^{u u}$ and $\epsilon^{v v}$ components rather than to $\epsilon^{u w}$ or $\epsilon^{w w}$. Hence, the $u w$ components of equivalent permittivity and permeability can often be ignored.

To compare rigorous calculations against those with the offdiagonal ( $u w)$ components in (14) omitted, we plotted in Fig. 3 the dispersion curve for the fundamental mode in a helical fiber as in [31], with $P=300 \mu \mathrm{m}$ and $R=400 \mu \mathrm{m}$, while the fiber core radius is $30 \mu \mathrm{m}$. Again, the FDFD method was used, but this time, when nonzero $u w$ components were present in $\epsilon$ and

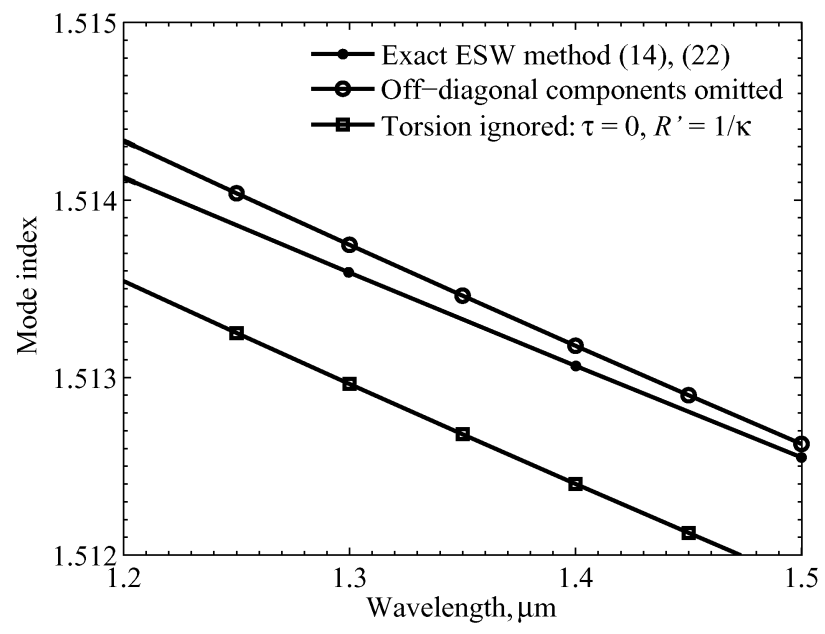

Fig. 3. Dispersion of a fundamental quasi- $E H_{11 u}$ mode in a helical fiber core similar to that in [31] (core radius $30 \mu \mathrm{m}$, numerical aperture $0.1, n_{\text {clad }}=$ 1.45 , twist parameters $P=300 \mu \mathrm{m}, R=400 \mu \mathrm{m}$ ) calculated rigorously; with the off-diagonal ( $u w$ ) components in (14) omitted; and with $\tau=0$, but $\kappa=R /\left(P^{2}+R^{2}\right)$ in (14) or, equivalently, $R^{\prime}=R+P^{2} / R$ and $P^{\prime}=0$.

$\mu$, as an eigenproblem in the frequency. The propagation constant $n_{\text {eff }}$ was an input parameter. This is not very convenient compared to solving an ordinary $n_{\text {eff }}$ eigenproblem, especially when material dispersion should be taken into account. Computation domain spanned $100 \mu \mathrm{m} \times 100 \mu \mathrm{m}$ and was discretized into $80 \times 80$ grid cells. Eight-cell-wide PMLs were constructed according to (18).

We see from this figure that omitting $u w$ components of $\epsilon$ and $\mu$ leads to nonnegligible deviation from rigorous results, but sometimes this can be an acceptable price for easier coding. One might further suggest that "the torsion effect ... can be neglected in helical fibers" [31], and use (20) or even (1) with the curvature radius $R^{\prime}=\kappa^{-1}=R+P^{2} / R$ (note that in [31], the $R$ term is incorrectly dropped out from this formula, leading to some nonphysical results in Section III therein). This amounts to setting $\tau=0$ in (14) while calculating $\kappa$ with nonzero $P$. Fig. 3 shows that this is not a good strategy when $P \approx R$, however, even for relatively large $P$ and $R$; besides, it brings no simplification to the structure of equivalent permittivity and permeability matrices.

\section{CONCLUSION}

Expressions (14a)-(14d) for an isotropic waveguide and (15) for an anisotropic one have been derived, valid for arbitrary curvature and torsion. Together with the PMLs given by (17), this enables rigorous full-vector modeling of very tight and lossy waveguide bends and twists with the finite-difference or finiteelement tools designed for straight waveguides; the only price is that even for waveguides consisting of physically isotropic homogeneous materials, the equivalent $\epsilon$ and $\mu$ are not scalars, but $3 \times 3$ matrices with components continuously varying with $v$ (or with both $u$ and $v$ for twisted anisotropic waveguides).

In the paper, we assumed the curvature and torsion of a waveguide be constant. Their variation with the longitudinal coordinate $w, \kappa=\kappa(w)$ and $\tau=\tau(w)$ prevents rigorous separation of variables in Maxwell's equations, but still it can be specified through (14) or (15) for the beam propagation or time-domain 
simulations of adiabatically slow transitions. To calculate abrupt bend transition losses, standard coupled-mode theory can be applied once the mode spectra of the adjoint waveguide sections are found.

Finally, a similar "equivalent straight wire" model can be developed with the use of the helical coordinates (10) orthogonalized at $v=R$ for curved electronic wires.

\section{REFERENCES}

[1] H. J. M. Bastiaansen, J. M. van der Keur, and H. Blok, "Rigorous, fullvectorial source-type integral equation analysis of circularly curved channel waveguides," IEEE Trans. Microw. Theory Tech., vol. 43, no. 2, pp. 401-409, Feb. 1995.

[2] H. J. M. Bastiaansen, H. E. Crayé, and H. Blok, "Source-type integral equation analysis of circularly curved channel waveguides in a multilayered background," IEEE Trans. Microw. Theory Tech., vol. 43, no. 7, pp. 1597-1604, Jul. 1995.

[3] S. Kim and A. Gopinath, "Vector analysis of optical dielectric waveguide bends using finite-difference method," J. Lightw. Techol., vol. 14, no. 9, pp. 2085-2092, Sep. 1996.

[4] W. W. Lui, C.-L. Xu, T. Hirono, K. Yokoyama, and W.-P. Huang, "Full-vectorial wave propagation in semiconductor optical bending waveguides and equivalent straight waveguide approximations," $J$. Lightw. Techol., vol. 16, no. 5, pp. 910-914, May 1998.

[5] N.-N. Feng, G.-R. Zhou, C. Xu, and W.-P. Huang, "Computation of full-vector modes for bending waveguide using cylindrical perfectly matched layers," J. Lightw. Techol., vol. 20, no. 11, pp. 1976-1980, Nov. 2002.

[6] K. Kakihara, N. Kono, K. Saitoh, and M. Koshiba, "Full-vectorial finite element method in a cylindrical coordinate system for loss analysis of photonic wire bends," Opt. Express, vol. 14, pp. 11128-11141, 2006.

[7] R. Pregla, "The method of lines for the analysis of dielectric waveguide bends," J. Lightw. Techol., vol. 14, no. 4, pp. 634-639, Apr. 1996.

[8] W. Pascher, "Modelling of rib waveguide bends for sensor applications," Opt. Quantum Electron., vol. 33, pp. 433-449, 2001.

[9] I. A. Goncharenko, S. F. Helfert, and R. Pregla, "Radiation loss and mode field distribution in curved holey fibers," Int. J. Electron. Commun., vol. 59, pp. 185-191, 2005.

[10] J. Ritter and F. Amdt, "Efficient FDTD/matrix-pencil method for the full-wave scattering parameter analysis of waveguiding structures," IEEE Trans. Microw. Theory Tech., vol. 44, no. 12, pp. 2450-2456, Dec. 1996.

[11] D. I. Paul, I. J. Craddock, and C. J. Railton, "Simple and accurate hybrid FDTD model of uniform waveguide bends," Electron. Lett., vol. 40, pp. 247-248, 2004.

[12] J. Koning, R. N. Rieben, and G. H. Rodrigue, "Vector finite-element modeling of the full-wave Maxwell equations to evaluate power loss in bent optical fibers," J. Lightw. Techol., vol. 23, no. 12, pp. 4147-4154, Dec. 2005.

[13] J. Dacles-Mariani and G. H. Rodrigue, "Study of optically induced effects due to bending and twisting using the vector finite-element method," J. Opt. Soc. Amer. B, Opt. Phys., vol. 23, pp. 1743-1751, 2006.

[14] D. Marcuse, "Field deformation and loss caused by curvature of optical fibers," J. Opt. Soc. Amer., vol. 66, pp. 311-320, 1976.
[15] D. Marcuse, "Influence of curvature on the losses of doubly clad fibers," Appl. Opt., vol. 21, pp. 4208-4213, 1982.

[16] M. Heiblum and J. H. Harris, "Analysis of curved optical waveguides by conformal transformation," IEEE J. Quantum Electron., vol. QE-11, no. 2, pp. $75-83$, Feb. 1975 .

[17] T. N. Trinh and R. Mittra, "Field profile in a single-mode curved dielectric waveguide of rectangular cross section," IEEE Trans. Microw. Theory Tech., vol. MTT-29, no. 12, pp. 1315-1318, Dec. 1981.

[18] A. Nicolet, F. Zolla, and S. Guenneau, "Modelling of twisted optical waveguides with edge elements," Eur. Phys. J. Appl. Phys., vol. 28, pp. 153-157, 2004

[19] A. Nicolet, A. B. Movchan, S. Guenneau, and F. Zolla, "Asymptotic modelling of weakly twisted electrostatic problems," C. R. Mecanique, vol. 334, pp. 91-97, 2006.

[20] R. A. Waldron, "A helical coordinate system and its applications in electromagnetic theory," Quart. J. Mech. Appl. Math., vol. 11, pp. 438-461, 1958.

[21] E. J. Post, Formal Structure of Electromagnetics. Amsterdam, The Netherlands: North-Holland, 1962, (reprinted by Dover, 1997).

[22] S. S. Klyamkin, "Electromagnetic field equations for the helically symmetric systems," (in Russian) Electrichestvo, pp. 74-77, 1988.

[23] M. Germano, "On the effect of torsion on a helical pipe flow," J. Fluid Mech., vol. 125, pp. 1-8, 1982.

[24] K. S. Yee, "Numerical solution of initial boundary value problems involving Maxwell's equations in isotropic media," IEEE Trans. Antennas Propag., vol. 14, no. 5, pp. 302-307, May 1966.

[25] S. D. Gedney and J. A. Roden, "Numerical stability of nonorthogonal FDTD methods," IEEE Trans. Antennas Propag., vol. 48, no. 2, pp. 231-239, Feb. 2000.

[26] Y. Liu, "Fourier analysis of numerical algorithms for the Maxwell equations," J. Comput. Phys., vol. 124, pp. 396-416, 1996.

[27] Advances in Computational Electrodynamics: The Finite-Difference Time-Domain MethodA. Taflove, Ed. Boston, MA: Artech House, 1998, ch. 5.

[28] D. M. Shyroki and A. V. Lavrinenko, "Perfectly matched layer method in the finite-difference time-domain and frequency-domain calculations," Phys. Stat. Sol. B, vol. 244, no. 10, pp. 3506-3514, 2007.

[29] D. M. Shyroki, "Efficient Cartesian-grid-based modeling of rotationally symmetric bodies," IEEE Trans. Microw. Theory Tech., vol. 55, no. 6, pp. 1132-1138, Jun. 2007.

[30] K. R. Hiremath, M. Hammer, R. Stoffer, L. Prkna, and J. Čtyroký, "Analytic approach to dielectric optical bent slab," Opt. Quantum Electron., vol. 37, pp. 37-61, 2005.

[31] Z. Jiang and J. R. Marciante, "Mode-area scaling of helical-core, dualclad fiber lasers and amplifiers using an improved bend-loss model," $J$. Opt. Soc. Amer. B, Opt. Phys., vol. 23, pp. 2051-2058, 2006.

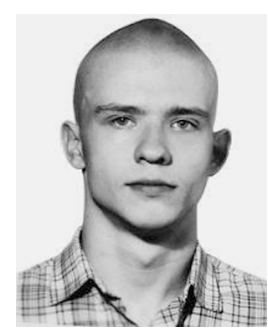

Dzmitry M. Shyroki was born in Minsk, Byelorussia, U.S.S.R., in 1981. He received the Specialist degree from Byelorussian State University, Minsk, Byelorussia, in 2004, and the Ph.D. degree from the Technical University of Denmark, Lyngby, Denmark, in 2008. 